\title{
Surface and smectic layering transitions in binary mixtures of parallel hard rods
}

\author{
Daniel de las Hera:* \\ Departamento de Fúsica Teórica de la Materia Condensada, \\ Universidad Autónoma de Madrid, E-28049 Madrid, Spain \\ Yuri Martínez-Ratón† \\ Grupo Interdisciplinar de Sistemas Complejos (GISC), Departamento de Matemáticas, \\ Escuela Politécnica Superior, Universidad Carlos III de Madrid, \\ Avenida de la Universidad 30, E-28911, Leganés, Madrid, Spain \\ Enrique Velascd \\ Departamento de Física Teórica de la Materia Condensada and Instituto de Ciencia de Materiales Nicolás Cabrera, \\ Universidad Autónoma de Madrid, E-28049 Madrid, Spain
}

(Dated: February 2, 2010)

\begin{abstract}
The surface phase behavior of binary mixtures of colloidal hard rods in contact with a solid substrate (hard wall) is studied, with special emphasis on the region of the phase diagram that includes the smectic A phase. The colloidal rods are modelled as hard cylinders of the same diameter and different lengths, in the approximation of perfect alignment. A fundamental-measure density functional is used to obtain equilibrium density profiles and thermodynamic properties such as surface tensions and adsorption coefficients. The bulk phase diagram exhibits nematic-smectic and smectic-smectic demixing, with smectic phases having different compositions; in some cases they are microfractionated. The calculated surface phase diagram of the wall-nematic interface shows a very rich phase behavior, including layering transitions and complete wetting at high pressures, whereby an infinitely thick smectic film grows at the wall via an infinite sequence of stepwise first-order layering transitions. For lower pressures complete wetting also obtains, but here the smectic film grows in a continuous fashion. Finally, at very low pressures, the wall-nematic interface exhibits critical adsorption by the smectic phase, due to the second-order character of the bulk nematicsmectic transition.
\end{abstract}

PACS numbers: 64.70.M-,61.30.Hn,61.20.Gy

\section{INTRODUCTION}

The wetting behavior of molecular smectic $(\mathrm{S})$ liquid

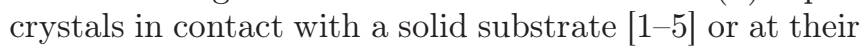
vapor-liquid crystal interfaces [6-10] has been an active research area since the 80 's. Partial or complete wetting behaviors of the $\mathrm{S}$ phase have been found when the isotropic (I) or nematic (N) phases are stable at bulk. Some liquid crystals exhibit a sequence of first-order layering transitions on decreasing the temperature slightly above the IS or NS bulk transition temperatures [1 10], indicating that the partial and complete wetting régimes can be mediated by a finite (or infinite) sequence of stepwise layer-adsorption transitions.

Usually the wetting behavior depends on specific interactions between the surface and the liquid crystal molecules, and molecular characteristics such as dipoles or length of alkyl chains. Depending on the strength of the interactions, solid substrates inducing orientational ordering of molecules may favor partial or complete wetting of the substrate by the $\mathrm{N}$ or $\mathrm{S}$ phases when the I

\footnotetext{
*Electronic address: daniel.delasheras@uam.es

† Electronic address: yuri@math.uc3m.es

‡Electronic address: enrique.velasco@uam.es
}

phase is stable at bulk, while those substrates promoting orientational disorder favor partial wetting behavior.

Freely-suspended smectic films, consisting of a few smectic layers surrounded by vapor (V), are formed by some liquid crystals and constitute another example of phase transitions induced by the presence of a surface. These films exhibit the so-called thinning transitions whereby the film thickness decreases stepwise as one or several layers (depending on the film heating rate) melt [1] 13 .

As usual in statistical mechanics, lattice models were the first to be applied to the study of the surface phase behavior of smectic liquid crystals. For example, a version of the Lebwohl-Lasher model, extended to include the smectic phase, was used to study the systematics of layering phenomena [14].

Density-functional theory (DFT) has been also successfully applied to the study of the surface phase behavior in liquid crystals adsorbed on solid substrates. The extension of the MacMillan theory to non-uniform phases with the inclusion of surface interaction potentials accounted for layering and thinning transitions [15, 16]. However, DFT models that incorporate repulsive interactions (reflecting molecular volume and shape) using either the Local- (LDA) [17] of Weighted-Density Approximation (WDA) [18], plus anisotropic attractive interactions via a mean-field approximation, turned out to be 
more realistic models for the calculation of surface phase diagrams. This is due to the fact that (i) the liquid crystal bulk phase behavior (e.g. values of coexistence densities and orientational order parameters) is better calculated from DFT, and (ii) interfacial properties, such as the width of the interface or the oscillatory behavior of the density profiles, are much better accounted for, due to the proper inclusion of pair correlations between particles.

For example, the wetting behavior of a smectic film in contact with an attractive wall has been successfully studied in Ref. [19], where the authors found complete or partial wetting by smectic depending on the strength of the external potential. A infinite (complete wetting) or finite (partial wetting) sequence of layering transitions was found, some of them ending in a prewetting line. Layering transitions at the V-I interface near the V-I-S triple point, and thinning transitions in freely-suspended smectic films, have been successfully studied using similar versions of DFT based on WDA and perturbation theory [20].

Finally, recent theoretical works have applied related models for hard rods in contact with a wall and/or confined between two walls. These studies were based on different approximations: Onsager with restricted orientations 21, 22], Onsager with Parsons-Lee rescaling and free orientations [23, 24] and also a WDA functional approximation 25, 26]. The surface phase diagram of a fluid of hard spherocylinders in contact with a single wall promoting different surface anchoring was analysed in Ref. [23]. In Refs. [25, 26] the surface phase diagram obtained for the confined fluid includes capillary nematization and smectization of the fluid, and a sequence of layering transitions of the confined smectic as the width of the slit pore is changed.

Practically all the experimental work on the wetting behavior of liquid crystals has been focused on onecomponent systems, the extension to mixtures being a pending issue. Adsorption phenomena in liquid crystal mixtures have a fundamental interest since bulk demixing transitions between two phases, at least one of them being smectic, would add much more complexity to the surface phase behavior. A recent theoretical work, based on Onsager theory, has analysed the phase behavior of the I-N interface of binary mixtures of hard spherocylinders [27]. Also, the substrate-isotropic interface of a mixture of hard parallelepipeds has been studied within the Zwanzig approximation [28]. However, it would be interesting to extend these studies to the high-pressure régime, where the smectic phase is stable.

One of the aims of the present work is to elucidate the role of the smectic phase in the interfacial phase behavior of binary mixtures. Recent theoretical models of mixtures of colloids (spherical or rod-like) and polymers [29 32] (based on the model proposed in Ref. [33] or on the recent fundamental-measure functional for hardsphere/hard-needle mixtures [34]) have shown that the entropic character of particle interactions, together with the coupling between species generated by the external surface potential, results in a rich phase behavior. For high polymer fugacities, partial wetting of the interface between the substrate and the fluid poor in colloidal particles by the fluid rich in colloidal particles was obtained. In the partial wetting régime, a sequence of up to four layering transitions was found. At lower fugacities complete wetting is reached via a first-order wetting transition (located below the critical point). These results were confirmed by Monte Carlo (MC) simulations [35].

Colloidal rod-like fluids and their mixtures are paradigmatic systems exhibiting liquid-crystal textures similar to those of molecular fluids, but the interaction between their components have an entropic origin due to shortranged repulsive forces. Intense experimental work on pure and mixed suspensions has been done in the last two decades, demonstrating this analogy [36]. Also, recent work has shown the importance of smectic layering in the kinetics of the NS phase transition in colloidal rods [37], confirming the analogy between molecular and colloidal fluids as regards the surface-enhanced smectic ordering near a bulk phase transition.

The aim of the present article is to theoretically analyse the surface phase diagram of a colloidal binary mixture of rods with the same breath but different lengths $L_{i}(i=1,2)$; in the following we use a length ratio $s=L_{2} / L_{1}=3$, with short species being labelled as 1 and the long species as 2. Particles interact through a hard repulsive potential and are constrained to be perfectly aligned along a nematic director, with their main axes perpendicular to a hard wall (W), thus simulating perfect homeotropic anchoring. This restriction, which considerably simplifies the model, is valid as long as one is only interested in the surface phase behavior of particles exhibiting a high degree of orientational order. The study concerns the wetting properties of these mixtures when a smectic film partially or completely wets the WN interface. Our theoretical tool is based on densityfunctional theory, more specifically on a recently proposed Fundamental-Measure Functional (FMF) for binary mixtures of parallel hard cylinders 38.

The impact of restricted orientations was analysed by Shundyak and van Roij in the context of the Onsager theory [39], using the Zwanzig model (discrete orientations). It was found to lead to spurious nematic phases with very high orientational order. Smectic phases were not analysed by Shundyak and van Roij but spurious smectic phases might well exist in Onsager theory. The parallel particle approximation implicit in the FMF approach is not expected to lead to any such anomalous phases, since the FMF theory contains a much better treatment of correlations, hence of the ordered phases.

As we will see later, the surface phase diagram of the model exhibits three different wetting behaviors depending on the value of pressure: (i) At high pressure $p$ we find complete wetting by smectic via an infinite sequence of layering transitions as the nematic binodal of the bulk NS transition is approached. These layering transitions 
end in corresponding surface critical points characterised by values of critical pressure $p_{c}^{(n)}, n=1,2, \ldots$ (ii) For sufficiently low pressure such that $p<p_{c}^{(n)}, \forall n$, wetting by the smectic film becomes continuous, with adsorption coefficients diverging logarithmically. And (iii) for pressures below the tricritical point, where the bulk NS transition changes from first to second order, we find critical adsorption by smectic. In this case a modified adsorption coefficient diverges logarithmically on approaching the second-order bulk NS transition. This divergence is a direct consequence of the NS bulk correlation length diverging at the transition.

The article is organized as follows. Sec. II is devoted to the presentation of the theoretical model and the numerical details relevant for the calculation of the bulk (Sec. IIA) and surface phase diagrams (Sec. IIB). In Sec. III we present the results obtained from numerical functional minimization. This section is divided into Sec. III A where the phase behavior of this particular mixture is described, Sec. IIIB, which contains a description of the layering transitions, and Sec. IIIC devoted to the study of the wetting behavior. Finally some conclusions are drawn in Sec. IV] Two appendices are included which contain mathematical details on the bifurcation analysis (Appendix A) and the derivation of the interfacial Gibbs-Duhem relation with composition and pressure as independent variables (Appendix B).

\section{THEORETICAL MODEL}

Our particle model consists of a binary mixture of parallel hard cylinders, with both species having the same diameter, $D_{1}=D_{2}=D$, chosen so as to set the ratio of transverse particle area and cylinder length squared of the short species to unity, i.e. $\pi D^{2} / 4 L_{1}^{2}=1$. This implies a particle aspect ratio of $L_{1} / D_{1}=0.89$. Since we choose a length ratio $s \equiv L_{2} / L_{1}=3$, the aspect ratio of the other particle is $L_{2} / D_{2}=2.66$. As densityfunctional theory and simulations show, a one-component fluid of parallel hard cylinders presents a phase sequence nematic-smectic-crystal, which is independent of the aspect ratio. The smectic phase of freely rotating hard spherocylinders is known to begin for aspect ratios $\gtrsim 4.1$, and we should expect a similar behavior for freely rotating hard cylinders. Since the phase behavior of a binary mixture of parallel particles with identical diameters but different lengths depends only on the ratio $L_{2} / L_{1}$, our model might describe a freely rotating binary mixture of cylinders with aspect ratios $L_{1} / D_{1}>4.1$ and $L_{2} / L_{1}=3$, both of which would have a smectic phase. Therefore, our choice guarantees that, in the one-component limits, the mixture would possess stable smectic phases at high enough pressure in the freely-rotating case.

\section{A. Bulk smectic phase}

A fundamental-measure density-functional theory for binary mixtures, in the version proposed in 38] and tested against MC simulations in [40], will be used in all calculations. We will consider a mixture which presents a non-uniform structure along the $z$ direction. The excess free-energy density reads

$$
\Phi L_{1}^{3}=n\left\{-\ln (1-\eta)+\frac{3 \eta}{1-\eta}+\frac{\eta^{2}}{(1-\eta)^{2}}\right\},
$$

where we drop the $z$-dependence for the sake of convenience and have defined the weighted densities

$$
\begin{aligned}
& n(z)=\frac{1}{2} \sum_{i}\left[\rho_{i}^{*}\left(z-\kappa_{i} / 2\right)+\rho_{i}^{*}\left(z+\kappa_{i} / 2\right)\right], \\
& \eta(z)=\sum_{i} \int_{z-\kappa_{i} / 2}^{z+\kappa_{i} / 2} \rho_{i}^{*}\left(z^{\prime}\right) d z^{\prime}
\end{aligned}
$$

with $\eta(z)$ the local packing fraction of the mixture. Index $i$ in all sums is assumed to run for $i=1,2$. We have defined the dimensionless densities $\rho_{i}^{*}(z)=\rho_{i}(z) L_{1}^{3}$, and $z$ coordinates are also in units of $L_{1}$. The $\kappa_{i}$ parameter is the particle length of species $i$ in the same units. Our choice for $L_{i}$ gives $\kappa_{1}=1$ and $\kappa_{2}=s=3$. The free-energy functional per unit area can be calculated as $\beta \mathcal{F} / A=\int d z\left[\Phi_{\text {id }}(z)+\Phi(z)\right]$, with $\beta^{-1}=k_{B} T$ the inverse thermal energy and

$$
\Phi_{\mathrm{id}}(z)=\sum_{i} \rho_{i}(z)\left[\ln \mathcal{V}_{i} \rho_{i}(z)-1\right]
$$

the ideal part of the free-energy density, where $\mathcal{V}_{i}$ is the thermal volume of species $i$. Now we specify for the smectic phase, which is the lowest symmetry phase considered in this work and has the property $\rho_{i}(z+k d)=\rho_{i}(z)$ (with $d$ the smectic period and $k \in \mathbb{Z}$ ). The pressure of the mixture can be calculated as

$$
\begin{array}{r}
\beta p L_{1}^{3}=d^{-1} \int_{0}^{d}\left\{\frac{n(z)}{1-\eta(z)}+\frac{3 n(z) \eta(z)}{[1-\eta(z)]^{2}}\right. \\
\left.+\frac{2 n(z) \eta(z)^{2}}{[1-\eta(z)]^{3}}\right\} d z .
\end{array}
$$

During the numerical minimization we have used the following constraints: (i) the value of the pressure $p$ is fixed, and (ii) the composition of the mixture, $x \equiv x_{1}=\rho_{1} / \rho$, is also set in advance. Here $\rho=\rho_{1}+\rho_{2}$ is the total mean density (calculated from the constant-pressure constraint), while $\rho_{i}=d^{-1} \int_{0}^{d} \rho_{i}(z) d z$ is the mean density of the $i$-th species. The Gibbs free-energy functional per particle, defined as

$$
\beta g\left[\rho_{1}, \rho_{2}\right]=\rho^{-1}\left\{d^{-1} \int_{0}^{d}\left[\Phi_{\mathrm{id}}(z)+\Phi(z)\right] d z+\beta p\right\},
$$


has been minimized with respect to the densities $\rho_{i}(z)$. We do this numerically by first discretising the densities, defining a grid with points $z_{k}=z_{0}+k \Delta(k=$ $0, \ldots, N)$, and then minimising the function $g\left(\boldsymbol{\rho}_{1}, \boldsymbol{\rho}_{2}\right)$ with respect to the components of the vectors $\boldsymbol{\rho}_{i}=$ $\left[\rho_{i}\left(z_{0}\right), \cdots, \rho_{i}\left(z_{N}\right)\right]$, and also with respect to $d$, using a conjugate-gradient algorithm. $N$ is the number of grid intervals. The width of the intervals was taken to be $\Delta / L_{1}=0.01$, and $N \Delta=m d$, where $m$ is the number of smectic periods within the minimization box. Varying $x$ between 0 and 1 and using the common-tangent construction for the function $\beta g(x)$, we have calculated the coexistence values for $x$ and $\rho$. Repeating the above procedure for different pressures, we obtained the demixing binodals.

When the NS transition is of the second order, one can use a bifurcation analysis to find the total packing fraction $\eta=\sum_{i} \rho_{i}^{*} \kappa_{i}$ and the smectic period $d$ at bifurcation (the local and total packing fractions are equal in the nematic phase. In the smectic phase the average of the local fraction $\eta(z)$ over one period gives the total packing fraction $\eta$ ). Also, with the aim to check the relative stability of the $\mathrm{S}$ with respect to the columnar (C) phase, we extended the bifurcation analysis to include the columnar symmetry. For this purpose we need to solve the following set of equations:

$$
\mathcal{H}(\mathbf{q}, \eta)=0, \quad \boldsymbol{\nabla} \mathcal{H}(\mathbf{q}, \eta)=0
$$

where the wave vectors $\mathbf{q}=(\mathbf{0}, q)$ and $\mathbf{q}=\left(\mathbf{q}_{\perp}, 0\right)$ are appropriate for the $\mathrm{S}$ and $\mathrm{C}$ symmetries, respectively. These equations have to be solved for the absolute minimum of $\mathcal{H}(\mathbf{q}, \eta) \equiv \operatorname{det}[H(\mathbf{q}, \eta)]$ as a function of $\mathbf{q}$, with $H(\mathbf{q}, \eta)$ a $2 \times 2$ matrix defined by the elements

$$
H(\mathbf{q}, \eta)=\left(\begin{array}{cc}
1-\rho_{1} \hat{c}_{11}(\mathbf{q}, \eta) & -\rho_{1} \hat{c}_{12}(\mathbf{q}, \eta) \\
-\rho_{2} \hat{c}_{12}(\mathbf{q}, \eta) & 1-\rho_{2} \hat{c}_{22}(\mathbf{q}, \eta)
\end{array}\right)
$$

with $\hat{c}_{i j}(\mathbf{q}, \eta)$ the Fourier transforms of the direct correlation functions calculated from the second functional derivatives of the free energy functional $\beta \mathcal{F}\left[\left\{\rho_{i}\right\}\right]$ with respect to $\rho_{i}(\mathbf{r})$ and $\rho_{j}\left(\mathbf{r}^{\prime}\right)$. Expressions for these functions and explicit results for the NS and NC spinodals can be found in Appendix A.

\section{B. Wall-fluid interface}

The aim is to calculate the equilibrium density profiles of the two species in the presence of a hard wall. The wall is located at $z=0$ and the long axes of cylinders are perpendicular to the wall. Thus, perfect homeotropic alignment of the nematic director is assumed. This model may apply to experimental systems where homeotropic anchoring is forced by surface treatment [41 44] or by the application of an external field (see [45] for an example on colloidal discs).
The values of the chemical potentials of the two components, $\mu_{i}$, will be fixed, which means that the conditions of the bulk fluid, far from the wall, will be specified in advance and maintained fixed during the minimisation. We minimise the grand potential functional per unit area,

$$
\frac{\Omega\left[\left\{\rho_{i}\right\}\right]}{A}=\frac{\mathcal{F}\left[\left\{\rho_{i}\right\}\right]}{A}+\sum_{i} \int\left[v_{i}(z)-\mu_{i}\right] \rho_{i}(z) d z
$$

with respect to the density profiles $\rho_{i}(z)$. The external potentials are defined by

$$
\beta v_{i}(z)=\left\{\begin{array}{l}
\infty, \quad z \leq L_{i} / 2, \\
0, \quad z>L_{i} / 2
\end{array} \quad i=1,2 .\right.
$$

To numerically implement the minimization we proceed by first choosing values for the pressure $p$ and the composition of the mixture at bulk, $x$, and from here calculating the chemical potentials $\mu_{i}$ and the dimensionless total density $\rho^{*}=\rho L_{1}^{3}$ at an infinite distance from the wall, using the following expressions, which apply to the bulk nematic phase:

$$
\beta p L_{1}^{3}=\frac{\rho^{*}(1+\eta)}{(1-\eta)^{3}}
$$

and

$$
\begin{aligned}
\beta \mu_{i} & =\ln x_{i}+\ln \left(\frac{\rho^{*}}{1-\eta}\right)+\frac{\eta(3-2 \eta)}{(1-\eta)^{2}} \\
& +\frac{\rho^{*}\left(4-3 \eta+\eta^{2}\right)}{(1-\eta)^{3}} \kappa_{i}, \quad i=1,2 .
\end{aligned}
$$

The implicit Eqn. (11) has to be solved iteratively to obtain $\rho^{*}$. In the minimisation the usual boundary conditions at a large distance $H$ from the wall, $\rho_{i}(H)=x_{i} \rho$, have to be imposed. $H$, the width of the minimization box, is chosen in such a way as to guarantee that the structure of the WN interface can be accommodated within the box and at the same time the boundary conditions are satisfied. Finally, the surface tension of the interface is calculated as $\gamma_{\mathrm{wN}}=\Omega\left[\left\{\rho_{i}^{(e)}\right\}\right] / A+p H$, with $\rho_{i}^{(e)}$ the equilibrium density profiles.

One of our aims is to obtain the wetting behaviour of the mixture when nematic conditions are fixed at bulk and the NS demixing transition is approached. This means that we need to calculate the surface tension of the WS interface for values of the chemical potentials $\mu_{i}$ corresponding to NS coexistence. Therefore $\mu_{i}$ can be calculated from Eqn. (12). However, if the bulk phase is a smectic, and consequently the density profiles are not uniform in bulk, the boundary conditions depend on the particular value of $H$ chosen, which considerably complicates the numerical minimization. To avoid this problem, we choose to define a symmetric box by using the follow- 
ing external potentials:

$$
\beta v_{i}(z)=\left\{\begin{array}{lc}
\infty, & z \leq L_{i} / 2, \\
0, & L_{i} / 2<z<H-L_{i} / 2, \\
\infty, & z \geq H-L_{i} / 2 .
\end{array}\right.
$$

We minimize $\Omega\left[\left\{\rho_{i}\right\}\right]$ with respect to $\rho_{i}(z)$ by choosing $H$ large enough to accommodate two WS interfaces. However, due to long-ranged commensuration effects generated by the confinement of a layered phase with period $d$ in a slit of width $H$, the minimized grand potential exhibits an oscillatory behavior as a function of $H$, with an asymptotically decaying amplitude. To overcome this problem, we defined the curve obtained from the local minima of $\Omega\left[\left\{\rho_{i}^{(e)}\right\}\right] / A$ and extrapolated to $H \rightarrow \infty$ to obtain the value of $2 \gamma_{\mathrm{ws}}$ (i.e. two times the surface tension of the WS interface).

To find the surface tension of the NS interface we followed a similar procedure: we defined a box of width $H$ with boundary conditions $\rho_{i}(0)=\rho_{i}(H)=\rho_{i}^{(\mathrm{N})}$ (the densities of the bulk nematic phase coexisting with smectic) at both ends of the box. Choosing an initial guess for $\rho_{i}(z), 0<z<H$ (close to the profiles of the coexisting bulk smectic phase), we minimized the grand potential to obtain $2 \gamma_{\mathrm{NS}}$ (i.e. two times the surface tension of the NS interface). Again the value of $H$ has to be large enough to accommodate two NS interfaces. Having the surface tensions of all the three different interfaces, one can study the wetting behavior of the system, which is discussed in Sec. IIIC]

Adsorption coefficients will also be used as a convenient measure of the wetting and adsorption properties of the WN interface. The adsorption coefficients of both species are defined as

$$
\Gamma_{i}=\int_{0}^{H}\left[\rho_{i}(z)-\rho_{i}^{(\mathrm{N})}\right] d z, \quad i=1,2 .
$$

In Appendix $B$ a derivation is presented of the interfacial Gibbs-Duhem relation expressed in terms of the independent variables $x$ and $p$. Using this equation, a relation between the derivative of $\gamma_{\mathrm{wN}}$ with respect to the composition variable $x$ and the adsorption coefficients can be obtained:

$$
\beta \frac{d \gamma_{\mathrm{wN}}}{d x}=\mathcal{U}(x, p)\left(\frac{\Gamma_{2}}{1-x}-\frac{\Gamma_{1}}{x}\right),
$$

where $\mathcal{U}(x, p)$, a function of bulk composition and pressure, is always positive if the binary mixture is stable against NN demixing. This relation has been tested (see Appendix B) to check for consistency of our numerical minimization procedure. Also, the sum rule relating the bulk pressure with the densities at the wall (contact theorem), $\beta p=\rho_{1}\left(L_{1} / 2\right)+\rho_{2}\left(L_{2} / 2\right)$, which is automatically satisfied by the functional, provides another check for numerical accuracy. For example, for a mixture with bulk pressure $\beta p L_{1}^{3}=1$ and composition $x=0.82$, we obtain $\left(\rho_{1}\left(L_{1} / 2\right)+\rho_{2}\left(L_{2} / 2\right)\right) L_{1}^{3}=0.994,0.997$ and 0.999 for values of the discretisation interval along the $z$ axis of $\Delta L_{1}^{-1}=0.0100,0.0050$ and 0.0025 , respectively (obviously, in the limit where $\Delta z \rightarrow 0$, the sum rule becomes exact).

\section{RESULTS}

This section is devoted to the presentation of the results obtained from our theoretical model. It is divided into three different sections. In Sec. III A we present and describe the main features of the bulk phase diagram. Secs. IIIB and IIC are devoted to the layering transitions and to the wetting behavior, respectively.

\section{A. Bulk phase diagram}

The bulk phase diagram of the binary mixture, shown in Fig. 1, has been calculated using bifurcation analysis and density-functional minimization, as described in Sec. IIA Two NS spinodals (dashed curves in Fig. 1), calculated from the bifurcation analysis, depart from the one-component limits $x=0$ and $x=1$ (where, as defined above, $x$ is the composition of the mixture as given by the fraction of short particles). The values of pressure in both spinodals increase as the composition of the mixture becomes farther from these limits, indicating that the two species cannot be easily accommodated into a smectic arrangement. The spinodal lines end in a tricritical point (filled circle) and a critical end point (filled square), respectively. Functional minimization indicates that the Gibbs free energy of the smectic phase is always a convex function of composition $x$ in the neighbourhood of (and above) these lines, which proves that the NS transition is of second order, with the smectic order parameter increasing from zero at the bifurcation. For pressures above the tricritical point but below the critical end point, the mixture segregates into a smectic phase rich in the long species and a nematic phase rich in the short species.

Two different smectic phases occur in the region of smectic stability. These phases are distinguished by the relative location of the density peaks of the two species. In the smectic phase labelled as $\mathrm{S}_{1}$ the profiles are in phase, with density peaks of the two species located at the same positions, which define the location of the smectic layers. In the phase called $\mathrm{S}_{2}$ the density profiles are out of phase, forming alternating smectic layers: this phase exhibits microfractionation [46 48]. The smectic mixtures with a higher fraction of species 1 (the short component) are denoted with a prime in Fig. 1. Examples of density profiles corresponding to these two smectic phases are shown in Figs. 2(a)-(d). Several regions of smectic coexistence exist in the mixture: $\mathrm{S}_{1}-\mathrm{S}_{2}$ in a narrow pressure interval between a critical and a triple 


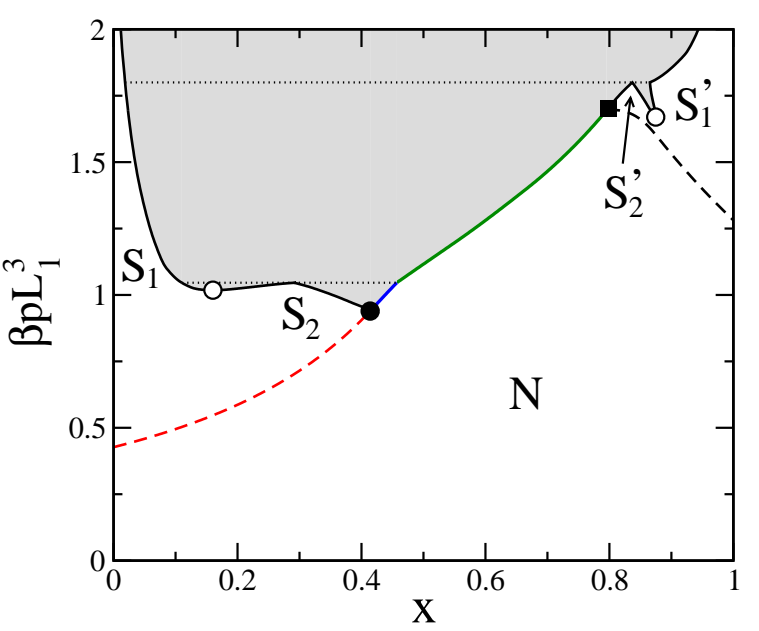

FIG. 1: (Colour online). Bulk phase diagram of the binary mixture of parallel hard cylinders in the reduced pressure $\beta p L_{1}^{3}$-composition $x$ plane (with $x=x_{1}$, the fraction of short particles). Dashed curves represent second-order NS transitions, while solid lines are the binodals of the NS or SS demixing transitions. The shaded area is the region of instability. Stability regions of nematic and different smectic phases are denoted by letters (see text). Colour lines indicate the conjectured wetting behaviour along the NS lines: critical adsorption (red), complete wetting by $\mathrm{S}_{2}$ smectic phase without layering transitions (blue), and complete wetting by $\mathrm{S}_{1}$ phase mediated by layering transitions (green). Circles: critical points. Square: critical end-point. Triangle: tricritical point.

point, $S_{1}^{\prime}-S_{2}^{\prime}$ in a corresponding interval between critical and triple points, and $\mathrm{S}_{1}-\mathrm{S}_{1}^{\prime}$ and $\mathrm{S}_{1}-\mathrm{S}_{2}^{\prime}$ coexistences at high pressure (triple points have been indicated by horizontal dashed lines in the figure).

We now comment on the possible stability of the columnar phase. A complete calculation of the stability of the columnar phase by free-energy minimisation is, at present, a highly difficult task. The difficulties stem from the computation of two-particle weighted densities [38]. Therefore, we have implemented a bifurcation analysis, which gives the conditions under which the nematic phase becomes unstable with respect to columnar-like fluctuations. As shown in Appendix $\mathrm{A}$, the NC spinodal, signalling the instability of the nematic phase against columnar-like fluctuations, is always located above the NS spinodal for all values of composition. This is an indication that at least part of the phases depicted in the phase diagram of Fig. 1 1 could be stable, and that the surface behaviour to be described below could represent the real surface behaviour of the model. However, one has to be cautious, since a first-order nematic-columnar and/or smectic-columnar phase transition could be greatly displaced with respect to the spinodal lines. Experimentally, rod-like colloidal particles always have some degree of polydispersity. Diameter polydispersity would tend to destabilise the columnar phase against the smectic phase, while breadth polydispersity would have the opposite ef-
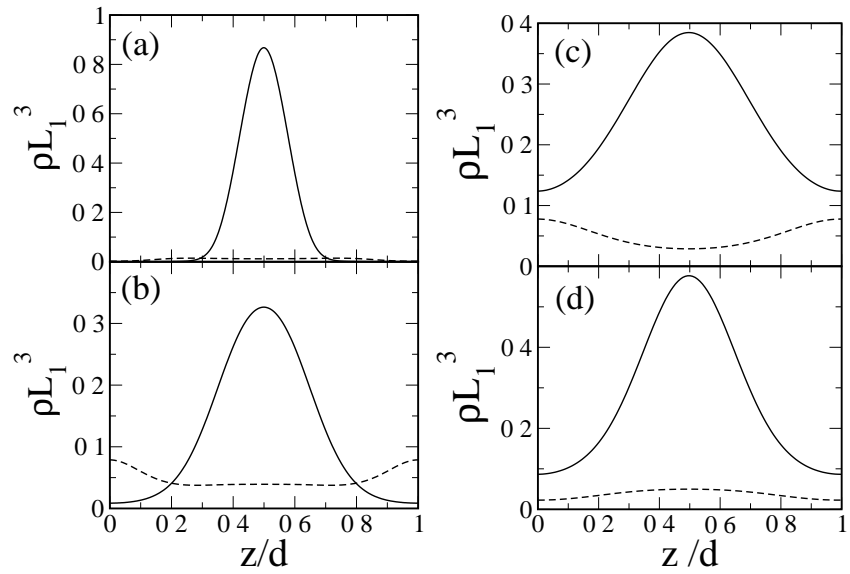

FIG. 2: Density profiles of (a) $\mathrm{S}_{1}$, (b) $\mathrm{S}_{2}$, (c) $\mathrm{S}_{2}^{\prime}$ (c), and (d) $\mathrm{S}_{1}^{\prime}$ phases in one smectic period. Values of smectic period are: (a) $d / L_{1}=3.495$, (b) 3.835, (c) 1.433 , and (d) 1.237 . In all figures solid and dashed curves correspond to species 2 and 1, respectively. Values of reduced pressure and composition, $\left(\beta p L_{1}^{3}, x\right)$, are: (a) $(1.20,0.06)$, (b) $(1.04,0.28)$, (c) $(1.75,0.83)$, and $(\mathrm{d})(1.75,0.88)$.

fect [49]. The final balance may depend on several effects in a delicate manner. Therefore, one has to be cautious until the following aspects are considered: (i) particle polydispersity in length and breadth, and (ii) full minimization of the density functional with respect to density profiles having columnar symmetry. We do not pursue this analysis here, which is left for future work.

\section{B. Layering transitions}

In this section, which constitutes the cornerstone of the present work, we present a detailed study of the layering transitions in the mixture. The stable bulk phase (in the region infinitely away from the wall) will be chosen to be a nematic phase, characterised by particular values of pressure and composition. We first consider the case where the pressure is given a value $\beta p L_{1}^{3}=1.30$ and the bulk composition $x$ is decreased from a high value close to unity. As the nematic branch of the $\mathrm{NS}_{1}$ binodal is approached, a sequence of layering transitions is found. At each of these transitions a new smectic layer, mostly composed of particles of the long species, appears through a first-order (interfacial) phase transition. This is illustrated in Fig. 3 where four equilibrium WN interfaces containing $0,1,2$ and 3 smectic layers composed (essentially) of particles of species 2 are shown. These structures will be denoted by $\mathrm{WN}_{i}$, with $i$ the number of adsorbed layers.

The structure of the $\mathrm{WN}_{0}$ interface is interesting. Right at the wall there is a mixture of highly localised long and short particles with similar densities. For increasing distance from the wall the density structure becomes much weaker [see Fig. 3(a)]. At the first $\left(\mathrm{WN}_{0^{-}}\right.$ 


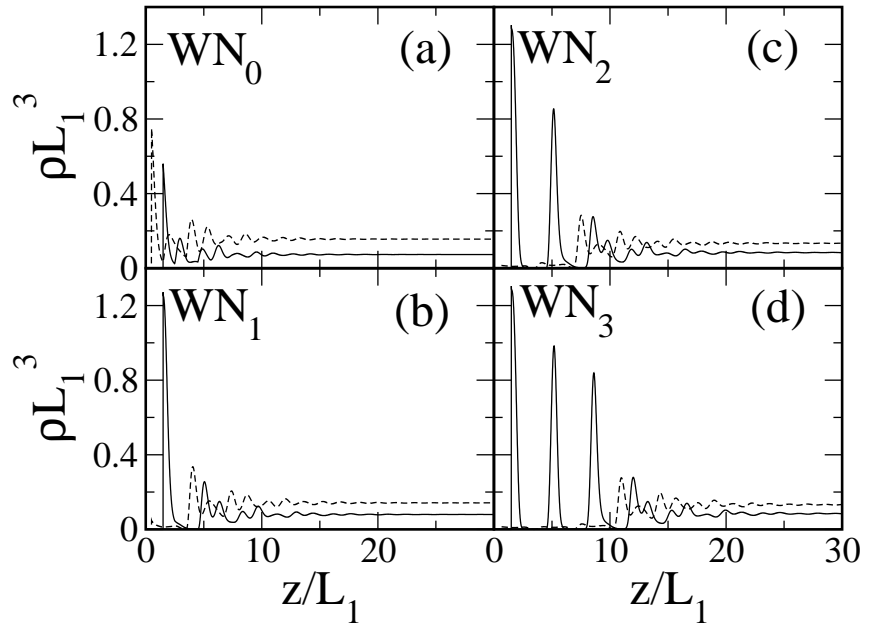

FIG. 3: Density profiles of species 1 (dashed line) and 2 (solid line) for $\beta p L_{1}^{3}=1.30$ and (a) $x=0.6800$, (b) 0.6400, (c) 0.61400 and (d) 0.6120 (coexistence value of composition is $\left.x_{\text {coex }}=0.6115\right)$. The symbol $\mathrm{WN}_{i}(i=1,2,3 \ldots)$ denotes the interfacial structure containing $i$ adsorbed layers.

$\mathrm{WN}_{1}$ ) layering transition, the wall becomes fully covered by long particles and a single very high density peak appears [Fig. 3(b)]. On further decreasing $x$, the system exhibits a sequence of phase transitions, $\mathrm{WN}_{i}-\mathrm{WN}_{i+1}$, each involving the addition of a further highly localised peak of the long particles. At $x=x_{\text {coex }}=0.6115$ (the composition of the bulk nematic phase coexisting with the $S_{1}$ phase at bulk), the wall is completely wet by the $S_{1}$ phase, which means that a macroscopically thick smectic film (consisting of an essentially infinite number of smectic layers) is interposed between the wall and the nematic phase. We have found up to 12 layering transitions as $x \rightarrow x_{\text {coex }}$ with $x>x_{\text {coex }}$. Access to higher-order layering transitions was not possible within the accuracy of our numerical procedure.

In Fig. 4 the behavior of the WN surface tension $\gamma=\gamma_{\mathrm{wN}}$, as a function of composition, is shown. The location of the $\mathrm{WN}_{i-1}-\mathrm{WN}_{i}$ layering transition is obtained from the intersection of the surface tensions corresponding to the two structures. The surface tension of the $\mathrm{WN}_{0}$ structure, shown in the inset, is somewhat peculiar: just before the $\mathrm{WN}_{0}-\mathrm{WN}_{1}$ layering transition, the surface tension exhibits a maximum. This behavior can be explained by resorting to Eqn. (15) and noting that the surface tension slope is exactly zero at $x^{*}=$ $\Gamma_{1} /\left(\Gamma_{1}+\Gamma_{2}\right)=\bar{\rho}_{1} /\left(\bar{\rho}_{1}+\bar{\rho}_{2}\right)$, where $\bar{\rho}_{i}=H^{-1} \int_{0}^{H} \rho_{i}(z) d z$, i.e. when the composition of the mixture at bulk coincides with its interfacial value. If $x>x^{*}$, i.e. when the interfacial composition is lower than the bulk value, the slope of the surface tension is positive, while the opposite occurs for $x<x^{*}$.

The adsorption coefficients $\Gamma_{i}$, defined in (14), are plotted in Fig. 5 as a function of $x$. As can be seen, $\Gamma_{2}$ abruptly increases at the layering transitions while $\Gamma_{1}$ abruptly decreases, i.e. the WN interface exhibits ad-

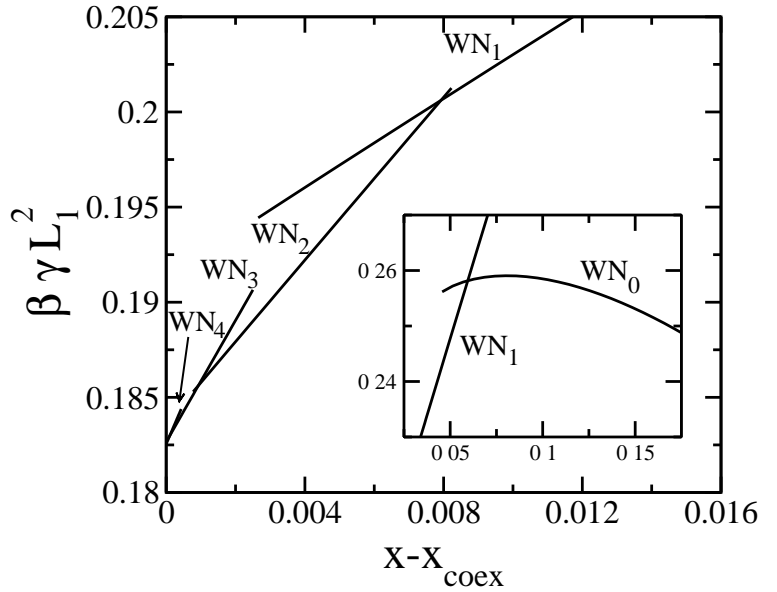

FIG. 4: Surface tension of the wall-nematic interface versus composition $x-x_{\text {coex }}$ for reduced pressure $\beta p L_{1}^{3}=1.3$. Symbols $W_{i}$ denote the different branches corresponding to wallnematic interfaces containing $i$ adsorbed smectic layers. The inset shows the surface tensions for the $\mathrm{WN}_{0}-\mathrm{WN}_{1}$ surface phase transition.

sorption of the long species and desorption of the small species. This is the natural interfacial path that connects a nematic phase located far from the wall, and rich in short particles, with a smectic film located next to the wall, and rich in long species, as $x \rightarrow x_{\text {coex }}$.

Repeating the same calculations, but at a lower value of pressure, $\beta p L_{1}^{3}=1.15$, we find that the first two layering transitions disappear, while the higher-order transitions $\mathrm{WN}_{i-1}-\mathrm{WN}_{i}$, with $i>2$, remain. Fig. 6(b) shows that, although the $\mathrm{WN}_{0}-\mathrm{WN}_{1}$ and $\mathrm{WN}_{1}-\mathrm{WN}_{2}$ transitions are absent, the adsorption coefficients significantly increase in the neighborhood of the transition points corresponding to a higher pressure. This behavior is consistent with the occurrence of critical points for the $\mathrm{WN}_{0}-$ $\mathrm{WN}_{1}$ and $\mathrm{WN}_{1}-\mathrm{WN}_{2}$ transitions at critical pressures in the interval $1.15<\beta p L_{1}^{3}<1.30$. Fig. 6)(a) shows a partial sequence of layering transitions involving up to 12 surface layers (the maximum number that our numerical scheme can deal with). It is reasonable to think that the layering transitions will continue up to the bulk transition in infinite number (complete wetting scenario).

A surface phase diagram that includes the first four layering transitions is shown in Fig. 77 The following trends can be extracted from the figure: (i) all layering transition curves approach the nematic binodal as the pressure is increased (for a number of layers $>5$ the curves are too close to the nematic binodal and are not visible in the figure); (ii) the critical points, where layering transitions terminate, move to lower pressures as the number of layers increases for $i \geq 2$. It is interesting to note that the critical point of the $\mathrm{WN}_{0}-\mathrm{WN}_{1}$ transition is located below that of the $\mathrm{WN}_{1}-\mathrm{WN}_{2}$ transition; this feature is related to the strong ordering of the $\mathrm{WN}$ interface just before the $\mathrm{WN}_{0}-\mathrm{WN}_{1}$ transition. In any case, layering transitions terminate at pressures where the bulk 


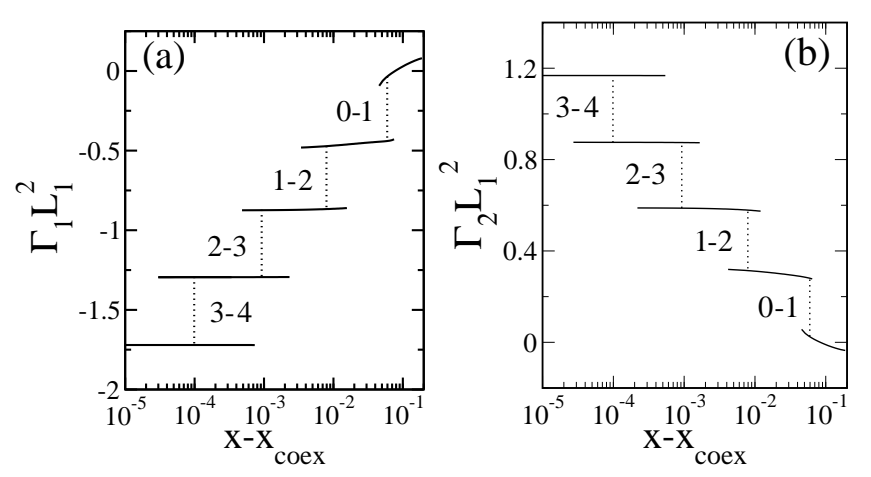

FIG. 5: Adsorption coefficients of (a) species 1, (b) species 2 , as a function of composition $x-x_{\text {coex }}$ (in logarithmic scale). Dotted lines indicate the transition points between two equilibrium interfaces. The value of the reduced pressure is $\beta p L_{1}^{3}=1.3$. Labels indicate the number of layers of the structures involved in each layering transition.

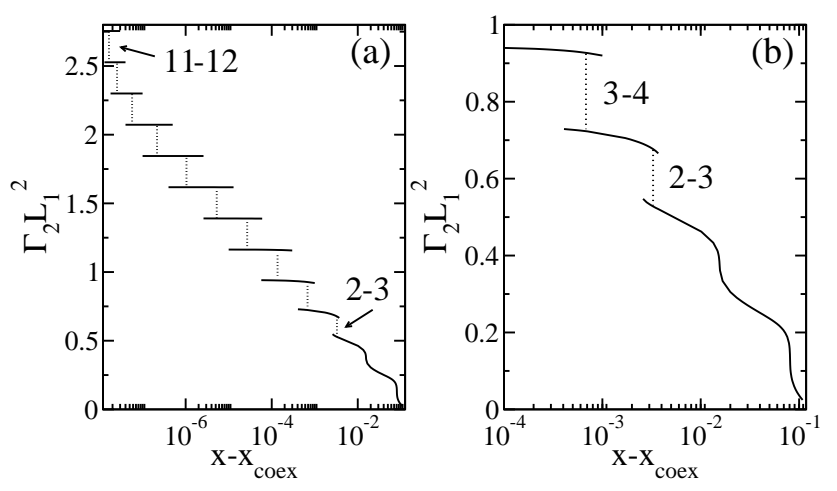

FIG. 6: (a) Adsorption coefficient of species 2 as a function of $x-x_{\text {coex }}$ (in logarithmic scale). Equilibrium structures are represented by continuous curves, while dashed vertical lines indicate layering transitions (metastability branches are not meant to be complete). (b) A zoom showing the first two layering transitions (in this case adsorption of the first two layers does not proceed via surface phase transitions). The pressure is fixed at $\beta p L_{1}^{3}=1.15$. Labels indicate the number of layers of the structures involved in each layering transition.

NS demixing transition becomes weak or disappears, i.e. when $\beta p L_{1}^{2} \simeq 1$.

We note that, depending on the wetting scenario for the $\mathrm{WS}_{2}^{\prime}$ interface, the layering transition curves could or could not continue above the $\mathrm{NS}_{2}^{\prime}$ spinodal; for example, the wetting régime could change to a partial wetting régime, similar to that found in Ref. [19]. Since interfacial calculations with a bulk smectic phase are difficult, we have not carried out this programme in the present work.

Next we briefly discuss the transition strength along the layering transition curves. In Fig. 8 the gap in the adsorption coefficient of species 2 at coexistence of the $\mathrm{WN}_{i-1}$ and $\mathrm{WN}_{i}$ structures, $\Delta \Gamma_{2}^{(i)}=\Gamma_{2}^{\left(\mathrm{WN}_{i}\right)}-\Gamma_{2}^{\left(\mathrm{WN}_{i-1}\right)}$, is plotted as a function of composition along the layering transition curves and for various indices $i$. The general

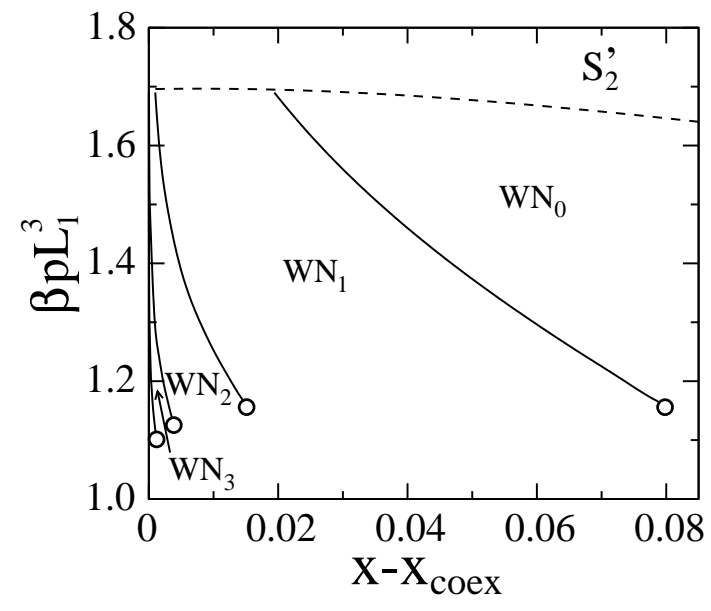

FIG. 7: Layering transitions (solid curves) between $\mathrm{WN}_{i-1}$ and $\mathrm{WN}_{i}$ interfacial structures (with $i$ the number of adsorbed layers) in the reduced pressure-composition plane. The critical points of the transitions are shown with open circles. Dashed line is the $\mathrm{NS}_{2}^{\prime}$ second order transition.

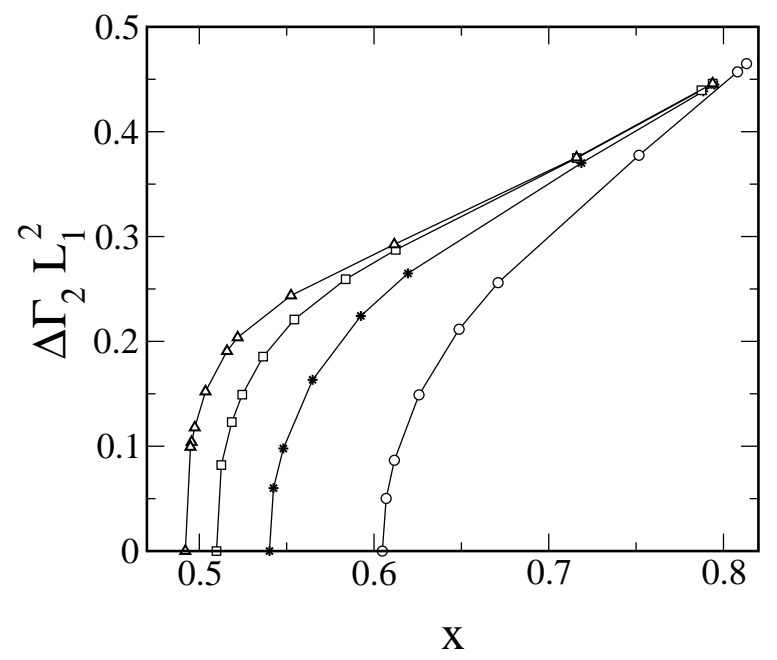

FIG. 8: Coexistence gap of the adsorption coefficient of the second species, $\Delta \Gamma_{2}^{(i)}$, at the $\mathrm{WN}_{i-1}-\mathrm{WN}_{i}$ layering transitions as a function of composition and for various indices $i$. Symbols refer to layering transitions $\mathrm{WN}_{0}-\mathrm{WN}_{1}$ (circles), $\mathrm{WN}_{1}-\mathrm{WN}_{2}$ (stars), $\mathrm{WN}_{2}-\mathrm{WN}_{3}$ (squares) and $\mathrm{WN}_{3}-\mathrm{WN}_{4}$ (triangles).

trend observed is that, as more layers get involved, the transition becomes stronger (i.e. the gap at coexistence is larger). As the index $i$ of the layering transition increases, the gap $\Delta \Gamma_{2}^{(i)}$ tends to saturate, corresponding to the fact that the additional layers adsorbed do not feel the effect of the wall and therefore contribute to the adsorption coefficient with a constant quantity.

We end this section with a comment on the origin of the layering transitions. As shown in [50], two-dimensional one-component hard-rod fluids in contact with a hard wall do not exhibit layering transitions even though the bulk transition is of first order [40]. This is also probably 
the case in the corresponding three-dimensional fluid, although we have not performed explicit calculations for the current model. Therefore, one tentative explanation for the phenomenology found in the mixture is that layering transitions are due to the entropic coupling between the two species mediated by the hard wall: next to the wall, where particle densities are high, both species compete for the gain in entropic volume. Just above the layering transition, a mixed layer packs less efficiently, and short particles are abruptly depleted from that region, with the subsequent abrupt increase in long particles. This conclusion would not be affected by the discovery of layering transitions in the calculations of Somoza et al. [19], who use particles with additional soft, temperaturedriven interactions; in this fluid the mechanism behind the layering transitions would be completely different.

A look at the structure of the density profiles of the $\mathrm{WN}_{0}$ interface [Fig. [3(a)] can help us understand this effect from a different perspective. The density maxima of the two species are clearly separated, due to the different lengths of the particles (density is maximum exactly at contact with the wall). However, the maxima of the bulk smectic phase are in phase $\left(\mathrm{S}_{1}\right.$ smectic). It is only because a sharp change in the interfacial structure occurs, via a first-order layering transition, that the interface can relax to a structure compatible with that in the bulk, i.e. with the correct relative phase. This mechanism operates even for structures $\mathrm{WN}_{i}$ with large $i$, when the effect of the wall is not crucial, because the density maxima of the two species, in the region between the already-formed smectic layers and the nematic, are always displaced one with respect to the other. At lower pressures, such that the bulk smectic phase is $\mathrm{S}_{2}$, there is no such incompatibility between the bulk structure and the structure imposed by the wall, and the layering transitions vanish.

\section{Wetting behavior}

To obtain a global picture of the wetting behavior of the mixture, we have concentrated on four different values of reduced pressure: $\beta p L_{1}^{3}=1.25$ and 1.30 (located above the bulk triple point, see Fig. 1), 1.00 (below the triple point and above the tricritical point), and 0.495 (below the tricritical point). In the first two cases we have found the phenomenology described in Sec. IIIB, i.e. an infinite sequence of layering transitions leading to complete wetting of the $\mathrm{WN}$ interface by the $\mathrm{S}_{1}$ phase. Using the procedures described in Sec. IIB, we have calculated the surface tensions of the $\mathrm{WN}, \mathrm{WS}_{1}$ and $\mathrm{NS}_{1}$ interfaces for $x=x_{\text {coex }}$, which are necessary to discuss the wetting behaviour. Their values are collected in Table I. As corresponds to complete wetting by the $\mathrm{S}_{1}$ phase, the surface tensions fulfill Young's law $\gamma_{\mathrm{ws}_{1}}=\gamma_{\mathrm{wN}}+\gamma_{\mathrm{Ns}_{1}}$ (the value of $\gamma_{\mathrm{wN}}$ at $x=x_{\text {coex }}$ can be computed by extrapolation of $\gamma_{\mathrm{WN}_{i}}$ with $i \rightarrow \infty$. In practice $i=32$ already gives enough accuracy to assess the wetting behaviour. Note that, in

\begin{tabular}{||cccccc||}
\hline \hline$\beta p L_{1}^{3}$ & $x_{\text {coex }}$ & $\gamma_{\mathrm{WS}_{\mathrm{j}}}^{*}$ & $\gamma_{\mathrm{NS}_{\mathrm{j}}}^{*}$ & $\gamma_{\mathrm{WN}}^{*}$ & $\gamma_{\mathrm{WS}_{\mathrm{j}}}^{*}+\gamma_{\mathrm{NS}}^{*}$ \\
\hline 1.300 & 0.61150 & 0.121166 & 0.061370 & 0.182535 & 0.182536 \\
1.250 & 0.58231 & 0.122408 & 0.055451 & 0.177859 & 0.177859 \\
1.000 & 0.43815 & 0.155570 & 0.001243 & 0.156822 & 0.156813 \\
0.495 & $0.10000^{*}$ & 0.181061 & - & 0.181061 & - \\
\hline \hline
\end{tabular}

TABLE I: Reduced surface tensions $\gamma^{*}=\beta \gamma L_{1}^{2}$ of the $\mathrm{WS}_{j}$, $\mathrm{WN}$ and $\mathrm{NS}_{j}$ interfaces for different values of the reduced pressure. Here $j=1,2$ depending on the nature of the smectic phase. ${ }^{*}$ indicates value at spinodal.

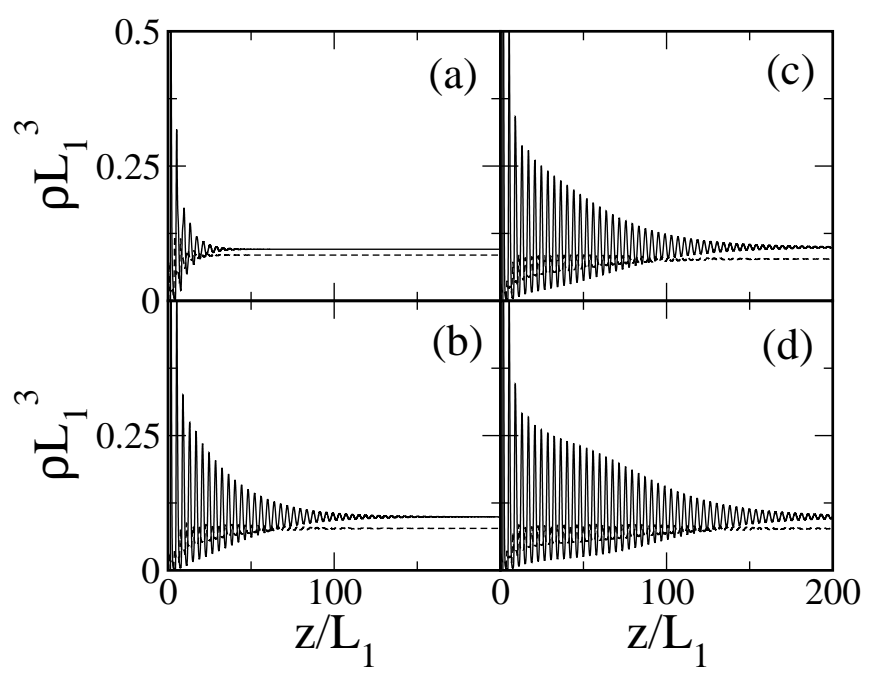

FIG. 9: Density profiles of species 1 (solid line) and 2 (dashed line) of the WN interface at bulk pressure $\beta p L_{1}^{3}=1.00$ and values of composition (a) $\Delta x=x-x_{\text {coex }}=3.2 \times 10^{-2}$, (b) $1.3 \times 10^{-3}$, (c) $3.2 \times 10^{-4}$ and (d) $6.4 \times 10^{-5}$. The first and, except in (a), the second density peaks are truncated due to the small scale of the vertical axis.

these cases, all the $\mathrm{WN}_{i}$ structures are metastable and can be stabilised, even at coexistence, under conditions of complete wetting, i.e. when the absolute free-energy minimum actually corresponds to $i=\infty$ ).

The wetting behavior for $\beta p L_{1}^{3}=1.00$ is similar to that found in [26] and [50] for one-component hard-rod systems: the thickness of the smectic film adsorbed at the WN interface grows continuously as $x \rightarrow x_{\text {coex }}$ and diverges at the bulk transition. This behavior is illustrated in Fig. 9, where four density profiles for values of composition very close to the bulk transition are shown. In this case layering transitions are completely absent; adsorption coefficients $\Gamma_{i}$ as a function of $x$ do not exhibit any discontinuity (Fig. 10), but diverge logarithmically as $x \rightarrow x_{\text {coex }}$. Young's law for complete wetting is also fulfilled within the numerical accuracy that could be achieved in this case (see Table凹). In this case the surface tension $\gamma_{\mathrm{NS}_{1}}$ is very small and is subject to higher uncertainties (the value of $\gamma_{\mathrm{WN}}$ was obtained by extrapolation to coexistence, $\left.x \rightarrow x_{\text {coex }}\right)$.

Next we discuss the equilibrium density profiles of the $\mathrm{WN}$ interface at a pressure $\beta p L_{1}^{3}=0.495$ (i.e. below 


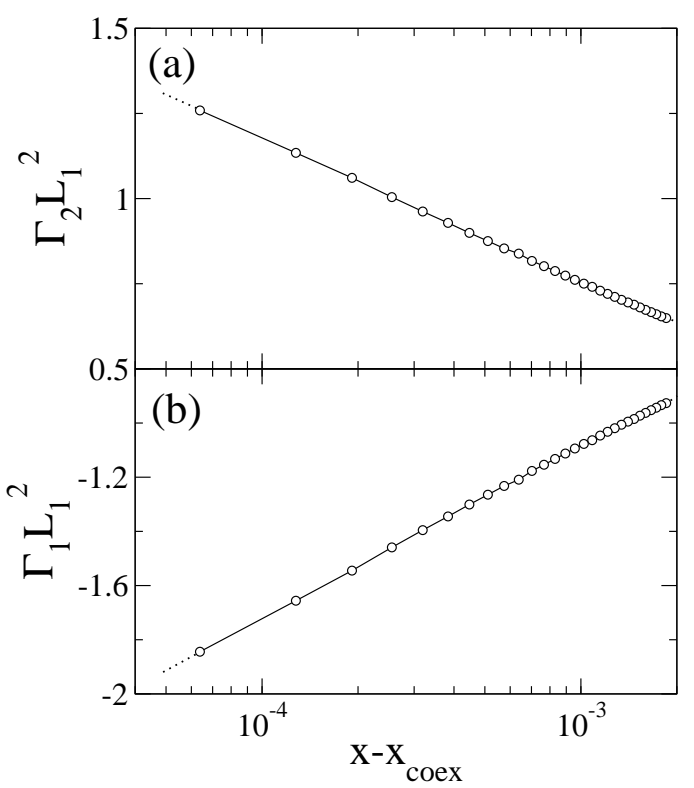

FIG. 10: Adsorption coefficients of species 2 (a) and 1 (b) as a function of composition (in logarithmic scale) near the wetting transition. The reduced bulk pressure is $\beta p L_{1}^{3}=1.0$.

the tricritical point) and, more specifically, the behavior of the adsorption coefficients as the bulk NS spinodal is approached. Let $x^{*}$ be the composition of the spinodal at a given pressure. Since the NS transition is of second order, we should find critical adsorption, similar to that occurring at the liquid-vapour critical point where the adsorption diverges logarithmically as dictated by meanfield theory. Far from the wall and close to the bulk spinodal, the WN interface exhibits oscillations with a period $d^{*}$ (the smectic period at bifurcation). Thus, the deviation of density profiles from their bulk values is better accounted for by the quantity $\left|\rho_{i}(z)-\rho_{i}\right|$ (the analogue of the order parameter in the anti-ferromagnetic Ising model), and it is convenient to define modified adsorption coefficients as

$$
\Gamma_{i}^{*}=\int_{0}^{H}\left|\rho_{i}(z)-\rho_{i}\right| d z .
$$

The behaviors of $\Gamma_{2}$ and $\Gamma_{2}^{*}$ as a function of $x-x^{*}$ are illustrated in Fig. 11] It can be seen from the figure that, while $\Gamma_{2}$ seems to reach a plateau as $x \rightarrow x^{*}$, the modified coefficient $\Gamma_{2}^{*}$ diverges logarithmically as predicted by mean-field theory for a critical adsorption phenomenon [51]. The value of the plateau is difficult to determine due to the huge values of $H$ required to accommodate the weakly damped interfacial oscillations that extend very far from the wall when $x \sim x^{*}$. The range of these oscillations is of the order of the smectic bulk correlation length, which diverges at $x=x^{*}$. Finally, we have checked that the system also exhibits critical adsorption in the neighbourhood of the $\mathrm{NS}^{\prime}$ spinodal curve (at higher pressure and composition).

We end this section with a discussion on the impact
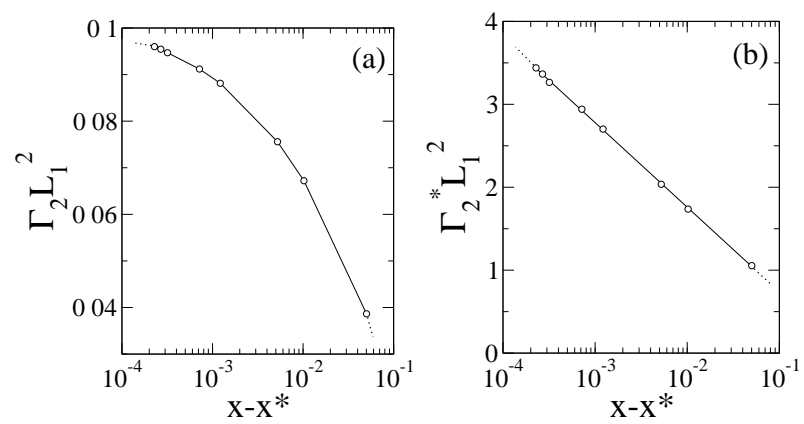

FIG. 11: The original (a) and modified (b) adsorption coefficients of second species as a function of bulk composition near the NS second order transition at $\beta p L_{1}^{3}=0.495$. In (a) the curve is a guide to the eye. In (b) the line is a logarithmic fit.

of the parallel-particle approximation on the wetting behaviour. Complete wetting (either continuous or via a sequence of layering transitions) of a hard wall by a binary mixture of hard particles is governed by two factors: (i) the effective entropic interactions between particles and the wall, and (ii) the distance of the bulk state point from the demixing binodal. Since both the free- and restrictedorientations models contain these two features, we only expect quantitative deviations between the two as far as the wetting behaviour is concerned. The situation with respect to the critical adsorption phenomenon is different, because this is due to the second-order character of the nematic-smectic transition, which may become weakly first order for small perturbations of particle orientations with respect to perfect alignment. In a model with free orientations the critical adsorption behaviour could be superseded by complete (continuous) wetting.

\section{CONCLUSIONS}

In this work we have studied the surface adsorption phenomena of a liquid-crystal colloidal mixture that has a stable smectic phase at moderate pressures. The mixture is described by means of a very simple model consisting of perfectly aligned hard particles, while the substrate is a hard wall inducing perfect homeotropic anchoring and nematic boundary conditions far from the wall are chosen. Even with these simple assumptions, the bulk and surface phase diagrams are so rich that we have concentrated only on the analysis of a single mixture with length ratio $s=L_{2} / L_{1}=3$. The theoretical tool used is a recently developed fundamental-measure density functional for mixtures of parallel cylinders 38.

We have found a bulk phase diagram with second order NS transitions at low pressures, followed by NS demixing above a tricritical point. In the low and high composition regions of the phase diagram two critical points exist, above which two smectic phases, one of them micro-fractionated, coexist. Coexistence is ended 
by corresponding triple points at higher pressures. At the highest pressures investigated smectic demixing is found, with each smectic rich in one of the species. A bifurcation analysis corroborates that the NC spinodal is always above the NS spinodal, but does not completely clarify the question about the absolute stability of the smectic against the columnar phases. In any case, we do not expect the NS, and possibly also the SS, demixing transitions to be preempted by the columnar phase at low pressures.

The surface phase diagram has three different wetting régimes. The first one, located below the tricritical point, exhibits critical adsorption as the composition of the bulk nematic phase approaches the NS spinodal. In the second régime, located approximately above the tricritical point and below the triple point (the exact boundaries would require further analysis), there exists complete wetting of the substrate by a smectic film whose thickness diverges logarithmically as $x \rightarrow x_{\text {coex }}$. Finally, the third régime is located above the triple point and is characterized by the presence of layering transitions that ultimately lead to complete wetting. A previous theoretical study of one-component hard-rod fluids using a density functional model [19] found layering phenomena in the semi-infinite system due to strong attractive interactions between the wall and the fluid particles. By contrast, layering transitions in hard-rod liquid-crystal mixtures adsorbed on a hard wall, as shown in the present study, is a direct consequence of the wall-mediated entropic interaction between the two species.

We expect that the present work serves as a starting point to initiate experimental studies on the surface phase behavior of liquid-crystal colloidal mixtures consisting of particles that interact through short-ranged repulsive forces, and having a stable bulk smectic phase. These experiments could be guided by the phenomenology found in the present study. Our future theoretical studies will analyse the adsorption phenomenology of films in the neighbourhood of the bulk triple point, a challenging problem that could provide further interesting phenomena.

\section{Acknowledgments}

We gratefully acknowledge financial support from Comunidad Autónoma de Madrid (Spain) under grants NANOFLUID, MOSAICO and S-0505/ESP-0299. This work is partly financed by grants FIS2005-05243-C0201, FIS2007-65869-C03-01, FIS2008-05865-C02-02 and FIS2007-65869-C03-C01 from Ministerio de Educación y Ciencia (SPAIN).

\section{Appendix A: Bifurcation analysis}

To implement the bifurcation analysis, we use the following expressions for the Fourier transforms of the direct correlation functions 38, 52]:

$$
\begin{aligned}
& \rho_{i} \hat{c}_{i j}(\mathbf{q}, \eta)=x_{i}\left\{2 l_{i j} \Psi_{0}\left(q_{\perp}\right) \chi\left(q_{\|} l_{i j}\right)\right. \\
& \left.+l_{i} l_{j} \Psi_{1}\left(q_{\perp}\right) \chi\left(q_{\|} l_{i} / 2\right) \chi\left(q_{\|} l_{j} / 2\right)\right\},
\end{aligned}
$$

where $l_{i}=L_{i} /\langle L\rangle, l_{i j}=\left(l_{i}+l_{j}\right) / 2,\langle L\rangle=\sum_{i} x_{i} L_{i}$, with $q_{\|}$and $q_{\perp}$ in units of $\langle L\rangle$ and $D / 2$, respectively. We have defined $\chi(x) \equiv \sin (x) / x$ and

$$
\begin{aligned}
\Psi_{0}(q)= & 4 y\left\{\frac{J_{1}(2 q)}{q}+2 y J_{0}(q) \frac{J_{1}(q)}{q}\right. \\
& \left.+y(1+2 y)\left[\frac{J_{1}(q)}{q}\right]^{2}\right\}, \\
\Psi_{1}(q)= & 4 y^{2}\left\{\frac{J_{1}(2 q)}{q}+2(1+2 y) J_{0}(q) \frac{J_{1}(q)}{q}\right. \\
& \left.+\left(1+6 y+6 y^{2}\right)\left[\frac{J_{1}(q)}{q}\right]^{2}\right\},
\end{aligned}
$$

with $y=\eta /(1-\eta)$ and $J_{n}(x)$ the n-th order Bessel function of first kind. The NS spinodal can be obtained by solving Eqs. (7) with $\mathbf{q}_{\perp}=\mathbf{0}, q_{\|}=q$, and

$$
\begin{aligned}
& \mathcal{H}(q, \eta)=1+\sum_{i} x_{i}\left\{2 \Psi_{0}(0) l_{i} \chi\left(q l_{i}\right)\right. \\
& \left.+\Psi_{1}(0) l_{i}^{2} \chi^{2}\left(q l_{i} / 2\right)\right\}-\left[\Delta \Psi_{0}(0) \chi(q \Delta l / 2)\right]^{2},
\end{aligned}
$$

where $\Delta l=l_{1}-l_{2}, \Delta^{2}=\left\langle l^{2}\right\rangle-1$ being the the polydispersity coefficient, with $\left\langle l^{2}\right\rangle=\sum_{i} x_{i} l_{i}^{2}$. For columnar symmetry $q_{\|}=0,\left|\mathbf{q}_{\perp}\right|=q$, and we find

$$
\mathcal{H}(q, \eta)=1+2 \Psi_{0}(q)+\Psi_{1}(q)+\Delta^{2}\left[\Psi_{1}(q)-\Psi_{0}^{2}(q)\right] .
$$

To search for a possible NN demixing scenarios, we need to solve $\mathcal{H}(0, \eta)=0$ for $\Delta$ as a function of $\eta$, which provides the NN spinodal:

$$
\Delta^{2}=\frac{1+2 \Psi_{0}(0)+\Psi_{1}(0)}{\Psi_{0}^{2}(0)-\Psi_{1}(0)}=\left(\frac{1}{\eta}-1\right)^{2} \frac{1+4 \eta+\eta^{2}}{7-2 \eta-\eta^{2}}
$$

For our particular mixture $\left(L_{2}=3 L_{1}\right)$, the pressure of the NN critical point is $\beta p L_{1}^{3} \approx 10$. This result shows the metastable character of the NN demixing against NS demixing (see Sec. IIIA).

In Fig. 12 the NS and NC spinodals, in the pressurecomposition plane, are shown. They never intersect and the former is always below the latter. This fact indicates that in the low-pressure region of the phase diagram, calculated in Sec. IIIA, the nematic and smectic phases could actually be stable against the columnar phase.

\section{Appendix B: Interfacial Gibbs-Duhem relation}

In this section we obtain the thermodynamic relation (15) involving the derivative of the WN surface tension 


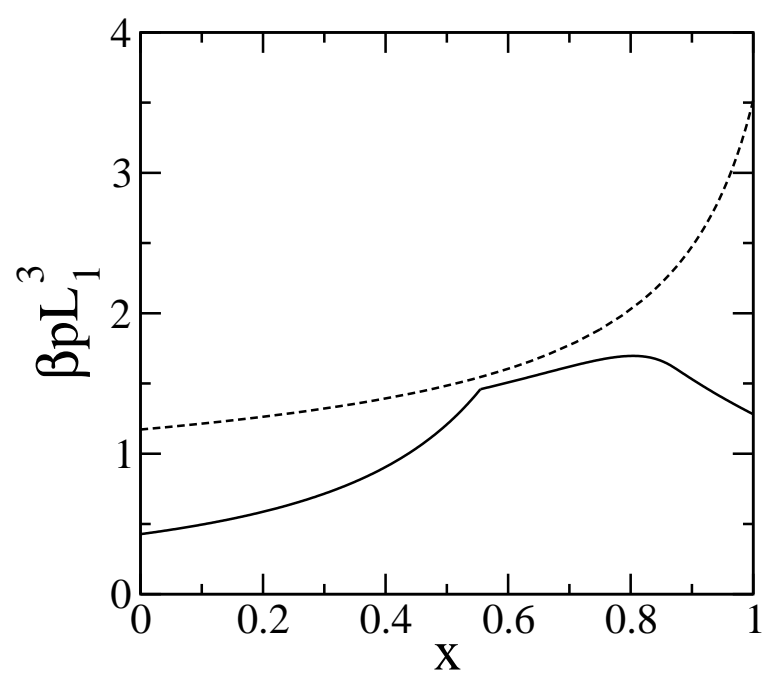

FIG. 12: NS (solid) and NC (dashed) spinodals in the reduced pressure-composition plane.

with respect to composition and the adsorption coefficients $\Gamma_{i}$. Starting from the interfacial Gibbs-Duhem relation $d \gamma=-\sum_{i} \Gamma_{i} d \mu_{i}$, we have

$$
\frac{d \gamma}{d x}=-\sum_{i} \Gamma_{i} \frac{d \mu_{i}}{d x}
$$

For fixed pressure $p_{0}$ the chemical potentials $\mu_{i}(x, \rho(x))$ are only functions of $x$ because $\rho(x)$ is defined implicitly through the constraint $p(x, \rho(x))=p_{0}$. Then:

$$
\frac{d \rho}{d x}=-\frac{\partial p / \partial x}{\partial p / \partial \rho}
$$

Taking into account that

$$
\frac{d \mu_{i}}{d x}=\frac{\partial \mu_{i}}{\partial x}+\frac{\partial \mu_{i}}{\partial \rho} \frac{d \rho}{d x}
$$

and using Eqs. (12), (111) and (B2), we finally find

$$
\beta \frac{d \mu_{i}}{d x}=\frac{(-1)^{i-1}}{x_{i}}-\frac{\Delta l \eta^{2}\left(7-2 \eta-\eta^{2}\right)\left(1-l_{i}\right)}{\left(1+4 \eta+\eta^{2}\right)(1-\eta)^{2}}
$$

which, after insertion in (B1), gives the final result (15), with

$$
\mathcal{U}(x, p)=1-\frac{\eta^{2}\left(7-2 \eta-\eta^{2}\right) \Delta^{2}}{\left(1+4 \eta+\eta^{2}\right)(1-\eta)^{2}}
$$

The function $\eta(x)$ in Eq. (B5) can be found from the constant-pressure constraint. Comparing Eqs. A6 and (B5), which contain the same and only density factor that can change sign, we conclude that $\mathcal{U}(x, P) \geq 0$ only when the mixture is stable against NN bulk demixing. To check for consistency of our numerical minimization procedure, we compare $d \gamma / d x$, as calculated from Eq. 15] [i.e. using the adsorption coefficients $\Gamma_{i}$ obtained from

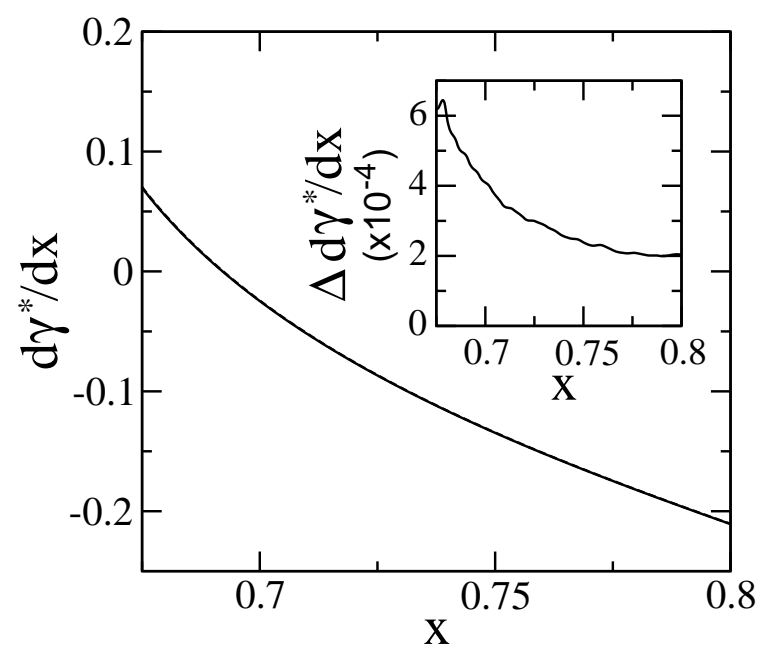

FIG. 13: Derivative of the reduced surface tension of the WN interface, $\gamma^{*}=\beta \gamma_{\mathrm{WN}} L_{1}^{2}$, with respect to composition $x$, obtained from numerical differentiation of the function $\gamma(x)$. In the inset the difference between this derivative and that obtained from Eqn. (15) is plotted (wiggles are due to noise in the numerical derivative). The value of the reduced pressure is $\beta p L_{1}^{3}=1.3$.

the equilibrium density profiles $\left.\rho_{i}^{(\mathrm{e})}\right]$ with the numerical derivative with respect to $x$ of the surface tension $\gamma$ obtained after minimization. Both results are plotted in Fig. 13 (for the WN interface) for $\beta p L_{1}^{3}=1.3$. As can be seen, both methods reproduce the same function with high accuracy, which demonstrates that our calculations are fully consistent. Note that the slope of the surface tension is equal to zero (and consequently $\gamma$ has a maximum as a function of $x$ ) for $x=\Gamma_{1} /\left(\Gamma_{1}+\Gamma_{2}\right)$ [see Eq. (15)], i.e. when the bulk composition is identical to the relative fraction of adsorption coefficient of species 1 .
[1] B. M. Ocko, Phys. Rev. Lett. 64, 2160 (1990).

[2] R. Lucht and Ch. Bahr, Phys. Rev. Lett. 78, 3487 (1997).

[3] T. Moses, Phys. Rev. E 64, 010702(R) (2001).

[4] T. Jin, G. P. Crawford, R. J. Crawford, S. Zumer, and D. Finotello, Phys. Rev. Lett. 90, 015504 (2003).

[5] Y. G. J. Lau, R. M. Richarson, and R. Cubitt, J. Chem.
Phys. 124, 234910 (2006).

[6] B. M. Ocko, A. Braslau, P. S. Pershan, J. Als-Nielsen, and M. Deutsch, Phys. Rev. Lett. 57, 94 (1986).

[7] P. S. Pershan, A. Braslau, A. H. Weiss, and J. AlsNielsen, Phys. Rev. A 35, 4800 (1987).

[8] R. Lucht, Ch. Bahr, G. Heppke, and J. W. Goodby, J. 
Chem. Phys. 108, 3716 (1998); R. Lucht, P. Marczuk, Ch. Bahr, and G. H. Findenegg, Phys. Rev. E 63, 041704 (2001).

[9] R. Lucht, Ch. Bahr, and G. Heppke, J. Phys. Chem. B 102, 6861 (1998); Phys. Rev. E 62, 2324 (2000).

[10] M. Fukuto, O. Gang, K. J. Alvine, B. M. Ocko, and P. S. Pershan, Phys. Rev. E 77, 031607 (2008).

[11] T. Stoebe, P. Mach, and C. C. Huang, Phys. Rev. Lett. 73, 1384 (1994)

[12] A. J. Jin, M. Veum, T. Stoebe, C. F. Chou, J. T. Ho, S. W. Hui, V. Surendranath, and C. C. Huang, Phys. Rev. E 53, 3639 (1996).

[13] E. I. Demikhov, V. K. Dolganov, and K. P. Meletov, Phys. Rev. E 52, R1285 (1995).

[14] Z. Pawlowska, G. F. Kventsel, and T. J. Sluckin, Phys. Rev. A 36, 992 (1987); Z. Pawlowska, T. J. Sluckin, and G. F. Kventsel, Phys. Rev. A 38, 5342 (1988).

[15] J. V. Selinger and D. R. Nelson, Phys. Rev. A 37, 1736 (1988).

[16] L. V. Mirantsev, Phys. Lett. A 205, 412 (1995).

[17] M. M. Telo da Gama, Mol. Phys. 52, 584 (1984); E. Martin del Rio, M. M. Telo da Gama, E. de Miguel and L. F. Rull, Phys. Rev. E 52, 5028 (1995).

[18] L. Mederos and D. E. Sullivan, Phys. Rev. A 46, 7700 (1992).

[19] A. M. Somoza, L. Mederos and D. E. Sullivan, Phys. Rev. Lett. 72, 3674 (1994); Phys. Rev. E 52, 5017 (1995).

[20] Y. Martínez-Ratón, A. M. Somoza, L. Mederos and D. E. Sullivan, Faraday Discuss. 104, 111 (1996).

[21] R. van Roij, M. Dijkstra and R. Evans, Europhys. Lett. 49, 350 (2000).

[22] R. van Roij, M. Dijkstra and R. Evans, J. Chem. Phys. 113,7689 (2000)

[23] D. de las Heras, L. Mederos, and E. Velasco, Phys. Rev. E 68, 031709 (2003).

[24] D. de las Heras, E. Velasco, and L. Mederos, J. Chem. Phys. 120, 4949 (2004).

[25] D. de las Heras, E. Velasco, and L. Mederos, Phys. Rev. Lett. 94, 017801 (2005).

[26] D. de las Heras, E. Velasco, and L. Mederos, Phys. Rev. E 74, 011709 (2006).

[27] K. Shundyak and R. van Roij, J. Phys.: Condens. Matter 13 (2001).

[28] L. Harnau and S. Dietrich, Soft Matter 3, 159 (2007).

[29] J. M. Brader, R. Evans, M. Schmidt, and H. Löwen, J. Phys.: Condens. Matter 14, L1 (2002).

[30] R. Evans, J. M. Brader, R. Roth, M. Dijkstra, M. Schmidt, and H. Löwen, Phil. Trans. R. Soc. Lond. A 359, 961 (2001).

[31] R. Roth, J. M. Brader, and M. Schmidt, Europhys. Lett. 63, 549 (2003).

[32] P. Bryk, J. Chem. Phys. 125, 204709 (2006).
[33] P. Bolhuis and D. Frenkel, J. Chem. Phys. 101, 9869 (1994).

[34] M. Schmidt, H. Löwen, J. M. Brader, and R. Evans, Phys. Rev. Lett. 85, 1934 (2000).

[35] M. Dijkstra and R. van Roij, Phys. Rev. Lett. 89, 208303 (2002).

[36] See for example: G. J. Vroege and H. N. W. Lekkerkerker, Rep. Prog. Phys. 55, 1241 (1992); M. Adams, Z. Dogic, S. L. Keller and S. Fraden, Nature 393, 349 (1998); H. Stark, J.-I. Fukuda and H. Yokoyama, Phys. Rev. Lett. 92, 205502 (2004); H. Maeda and Y. Maeda, Phys. Rev. Lett. 90, 018303 (2003).

[37] Z. Dogic, Phys. Rev. Lett. 91, 165701 (2003).

[38] Y. Martínez-Ratón, J. A. Capitán and J. A. Cuesta, Phys. Rev. E 77, 051205 (2008)

[39] K. Shundyak and R. van Roij, Phys. Rev. E 69, 041703 (2004).

[40] J. A. Capitán, Y. Martínez-Ratón and J. A. Cuesta, J. Chem. Phys. 128, 194901 (2008)

[41] K. E. Vaughn, M. Sousa, D. Kang, and C. Rosenblatt, Appl. Phys. Lett. 90, 194102 (2007).

[42] Y. Yi, G. Lombardo, N. Ashby, R. Barberi, J. E. Maclennan, and N. A. Clark, Phys. Rev. E 79, 041701 (2009).

[43] D. Zhao, W. Huang, H. Cao, Y. Zheng, G. Wang, Z. Yang and H. Yang, J. Phys. Chem. B 113, 2961 (2009).

[44] T. Beica, R. Moldovan, I. Zgura, S. Frunza, and M. Poterasu, J. Optoelectron. Adv. Mat. 8, 1512 (2006).

[45] K. Zhao, C. Harrison, D. Huse, W. B. Russel and P. M. Chaikin, Phys. Rev. E 76, 040401 (2007).

[46] T. Koda and H. Kimura, J. Phys. Soc. Japan 63, 984 (1994).

[47] G. Cinacchi, E. Velasco and L. Mederos, J. Phys.: Conden. Matter 16, S2003 (2004).

[48] G. Cinacchi, Y. Martinez-Raton, L. Mederos and E. Velasco, Mol. Cryst. Liq. Cryst. 465, 121 (2007).

[49] A. M. Bohle, R. Holyst and Thomas Vilgis, Phys. Rev. Lett. 76, 1396 (1996).

[50] Y. Martínez-Ratón, Phys. Rev. E 75, 051708 (2007).

[51] The bifurcation point for $\beta p L_{1}^{3}=0.495$ is at $x^{*}=0.10000$ as obtained numerically from Eqns. (7) and (A4). The data for $\Gamma_{2}^{*}$ in Fig. 10 are very sensitive to $x-x^{*}$ close to the bifurcation point, especially when plotted in logarithmic scale. An unwanted consequence of this is that the first two values in Fig. 10 exhibit a slight curvature if the above value of $x^{*}$ is used. This situation is improved if $x^{*}$ is obtained from a fit to the function $\Gamma_{2}^{*}=-a \log \left(x-x^{*}\right)-b$, with $a, b$ and $x^{*}$ free parameters. The fit gives $x^{*}=0.09987$. The data plotted in the figure were obtained using this modified value.

[52] Y. Martínez-Ratón and J. A. Cuesta, Mol. Phys. 107, 415 (2009). 